\section{Stars seen forming in a far-off galaxy}

Astronomers have seen their best glimpse yet of stars forming in the early Universe.

The ALMA radio telescope in Chile explored the SDP.81 galaxy, which is 3.6 billion parsecs ( 11.7 billion light years) away from Earth. Its light was magnified and distorted by the gravitational pull of another galaxy between it and Earth, but a model developed by Yoichi Tamura of the University of Tokyo and his team corrected the distortions. Their images reveal many cold clouds of dust and gas that are driving a rapid rate of star formation.

Several research teams have analysed the ALMA data to characterize other aspects of this galaxy.

Publ. Astron. Soc. Japan http://doi.org/5bv (2015)

\section{APPLIED PHYSICS}

\section{Evaporation} drives engine

An engine fuelled only by water evaporation can power a miniature car and lights.

Ozgur Sahin at Columbia University in New York and his colleagues applied bacterial spores to thin plastic strips. The spores absorb and release water with changes in relative humidity, so the strips curl and straighten. The team stacked the strips and formed them into a water-containing engine so that the strips were exposed to recurring periods of high and low humidity, acting like oscillators to power the engine. When attached to a generator, the engine powered light-emitting diodes. A rotary version attached to two pairs of wheels (pictured, left) pushed

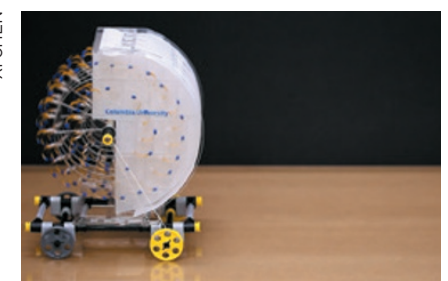

a 100-gram car forwards

(pictured, right).

The engine could be used in devices in areas that have scarce electricity, the authors say. Nature Commun. 6, 7346 (2015)

\section{BEHAVIOURAL ECOLOG}

\section{Angry spiders spin long-lived colonies}

Animal personality can influence the lifespan of whole ecological communities.

Female Anelosimus studiosus spiders create large webs shared by multiple females of this species and by many other types of spider. Jonathan Pruitt and Andreas Modlmeier of the University of Pittsburgh in Pennsylvania started colonies with either two docile spiders or two aggressive ones. Docile females allowed many kinds of spider to colonize and scavenged the other spiders' prey. Aggressive ones caught their own prey and fought off competing and predatory spiders. All of the communities collapsed in the end, but those founded by aggressive females lasted up to three years longer.

Communities founded by docile spiders reached a similar destabilizing species composition to aggressive spiders' colonies, but did so at a faster rate, making the docilefounded colonies shorter lived. J. Anim. Ecol. http://doi.org/5bt (2015)

\section{GENOME EDITING}

\section{CRISPR system} controlled by light

A popular genome-editing technique has been modified so researchers can use light to control when editing occurs and in which cells.

To better control how the CRISPR-Cas 9 system cuts

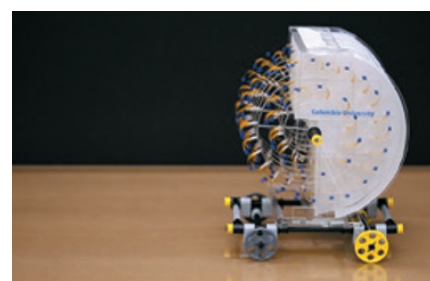

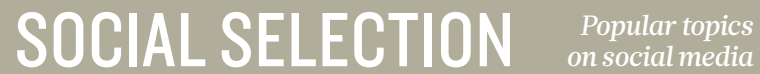

\title{
Unreliable dietary data under fire
}

Much of the data on personal eating habits - which form the basis of many national dietary guidelines - are unreliable and misleading, according to a report in Mayo Clinic Proceedings that drew attention on social media. The authors argue that the widely used survey method of asking people to recall their food and drink intake is so inaccurate that any conclusions based on such data should be called into question. "Must read paper in nutrition! At what point does inaccurate data become worse than no data?" tweeted Dylan MacKay, a human-nutrition researcher at the University of Manitoba in Winnipeg, Canada. But Brenda Davy, a clinical nutritionist at Virginia Polytechnic Institute and State University in Blacksburg, writes in an accompanying editorial that whereas self-reports are imperfect, they have helped to uncover vital nutritional links, such as that between fat intake and heart disease.

Mayo Clin. Proc. http://doi.org/5dd; http://doi.org/5df (2015)

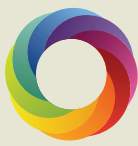

Based on data from altmetric.com. Altmetric tracks and compiles online attention around research outputs such as papers, books and data sets.
SNATURE.COM

For more on popular papers: go.nature.com/necdhq and alters DNA sequences, Moritoshi Sato and his team at the University of Tokyo in Japan split the Cas9 DNAcutting enzyme in two and fused each part to a protein. These proteins join together when hit with blue light, reactivating Cas9. They found that the modified enzyme could still edit the genome accurately in human cells in the lab. The frequency of edits increased with longer light exposures and no editing was seen in cells kept in the dark.

The team also shone patterns of light onto the cells and found that genome editing occurred along the same patterns. The technique could help to improve the precision of CRISPR, the authors say.

Nature Biotech. http://dx.doi. org/10.1038/nbt.3245 (2015)

\section{REGENERATIVE BIOLOGY}

\section{Mammary ducts made in a dish}

Cells from human mammary glands develop in the lab into structures that look and behave like milk-producing ducts in the breast.

A team led by Christina Scheel at the Helmholtz Center for Health and Environmental Research in Munich, Germany, obtained mammary gland tissue from healthy women undergoing surgery and isolated cells that line the ducts of the glands. The team suspended individual cells in a 3D flexible collagen gel, added growth factors, and found that the cells developed into structures that resembled the key functional units of the mammary gland. Some of the structures even contracted in the same way as ducts do during lactation.

Cells developed into ductlike structures only when they were grown in collagen gels that were not physically attached to the culture dish, suggesting that a flexible matrix is needed for normal growth of this tissue. Development http://dx.doi. org/10.1242/dev.123554 (2015)

\section{ONATURE.COM}

For the latest research published by Naturevisit:

www.nature.com/latestresearch 